\title{
Brain Abscess Caused by Pulmonary Arteriovenous Fistulas without Rendu-Osler-Weber Disease
}

\author{
Hiroyuki Kawano ${ }^{1,3}$, Teruyuki Hirano ${ }^{3}$, Koichi Ikeno ${ }^{1}$, Isao Fuwa ${ }^{2}$ and Makoto Uchino ${ }^{3}$
}

\begin{abstract}
A 52-year-old man was admitted with a brain abscess in the left basal ganglia. He had a paradoxical brain embolic mechanism owing to a pulmonary arteriovenous fistula (PAVF) and was diagnosed as having a rightto-left shunt by transesophageal echocardiography (TEE) and transcranial color Doppler (TCD) with saline contrast medium. We determined that the brain abscess was caused by the PAVFs without Rendu-OslerWeber disease. TEE and TCD with saline contrast medium were very useful for identifying the presence of the right-to-left shunt that caused the brain abscess.
\end{abstract}

Key words: pulmonary arteriovenous fistula, brain abscess, right-to-left shunt, transcranial color Doppler, transesophageal echocardiography

(Inter Med 48: 485-487, 2009)

(DOI: 10.2169/internalmedicine.48.1678)

\section{Introduction}

Rendu-Osler-Weber (ROW) disease is characterized by multiple dermal, mucosal and visceral telangiectasias that are associated with recurrent bleeding and pulmonary arteriovenous fistulas (PAVFs) (1). PAVFs may be congenital or acquired, solitary or multiple, microscopic or massive. The most prominent complications associated with PAVFs are neurologic events, including transient ischemic attacks, stroke, brain abscesses, and seizures (2). A right-to-left pulmonary shunt may provide access for the septic emboli to bypass the natural pulmonary capillary filter and cause brain abscesses. In a previous study, brain abscesses were reported to occur commonly in patients with pulmonary arteriovenous malformations in hereditary hemorrhagic telangiectasia (3). Here, we present a patient with a brain abscess associated with an isolated PAVF without ROW disease.

\section{Case Report}

A 52-year-old man was admitted to our hospital due to cough, fever, and headache without nausea. He had no significant past or family history of respiratory discomfort, bleeding tendencies, or capillary dilatation. On admission, the patient was alert and oriented. He had no cyanosis and no clubbing, but he had a fever of $38.5^{\circ} \mathrm{C}$ and neck stiffness. No neurological deficits were noted. No murmurs were heard over the chest. Laboratory data on admission showed leukocytes of $13,700 / \mathrm{mm}^{3}$ and C-reactive protein of 0.05 $\mathrm{mg} / \mathrm{dL}$, but no polycythemia. Chest X-ray films showed a nodular lesion in the lower lobe of the left lung (Fig. 1A). Computed tomography (CT) of the brain showed a low density area in the left basal ganglia (Fig. 1B). On the contrast enhanced T1-weighted image, the mass had ring enhancement and surrounding edema (Fig. 1C). The cerebrospinal fluid was mildly clouded, with an opening pressure of 140 $\mathrm{mmH}_{2} \mathrm{O}$, cells $3,397 / \mathrm{mm}^{3}$ (monocytes/neutrophils, 10/3,387), glucose $32 \mathrm{mg} / \mathrm{dL}$, and protein $128 \mathrm{mg} / \mathrm{dL}$. The patient was diagnosed as having a brain abscess, and antibiotics (ceftriaxone, clindamycin, ampicillin) were given. However, the brain abscess did not decrease in size. Therefore, the brain abscess was aspirated on the 10th day, and the aspirate was purulent material with Streptococcus intermedius. Since his arterial blood gases on room air showed hypoxemia without $\mathrm{PaCO}_{2}$ elevation $\left(\mathrm{PaO}_{2} 67.4\right.$ and $\left.\mathrm{PaCO}_{2} 36.8 \mathrm{mmHg}\right)$, he appeared to have a right-to-left shunt and a hematogenous source. A transesophageal echocardiogram (TEE) showed air

${ }^{1}$ Department of Neurology, Arao Municipal Hospital, Arao, ${ }^{2}$ Department of Neurosurgery, Arao Municipal Hospital, Arao and ${ }^{3}$ Department of Neurology, Graduate School of Medical Science, Kumamoto University, Kumamoto

Received for publication September 9, 2008; Accepted for publication November 27, 2008

Correspondence to Dr. Hiroyuki Kawano, hkawanoo@gmail.com 

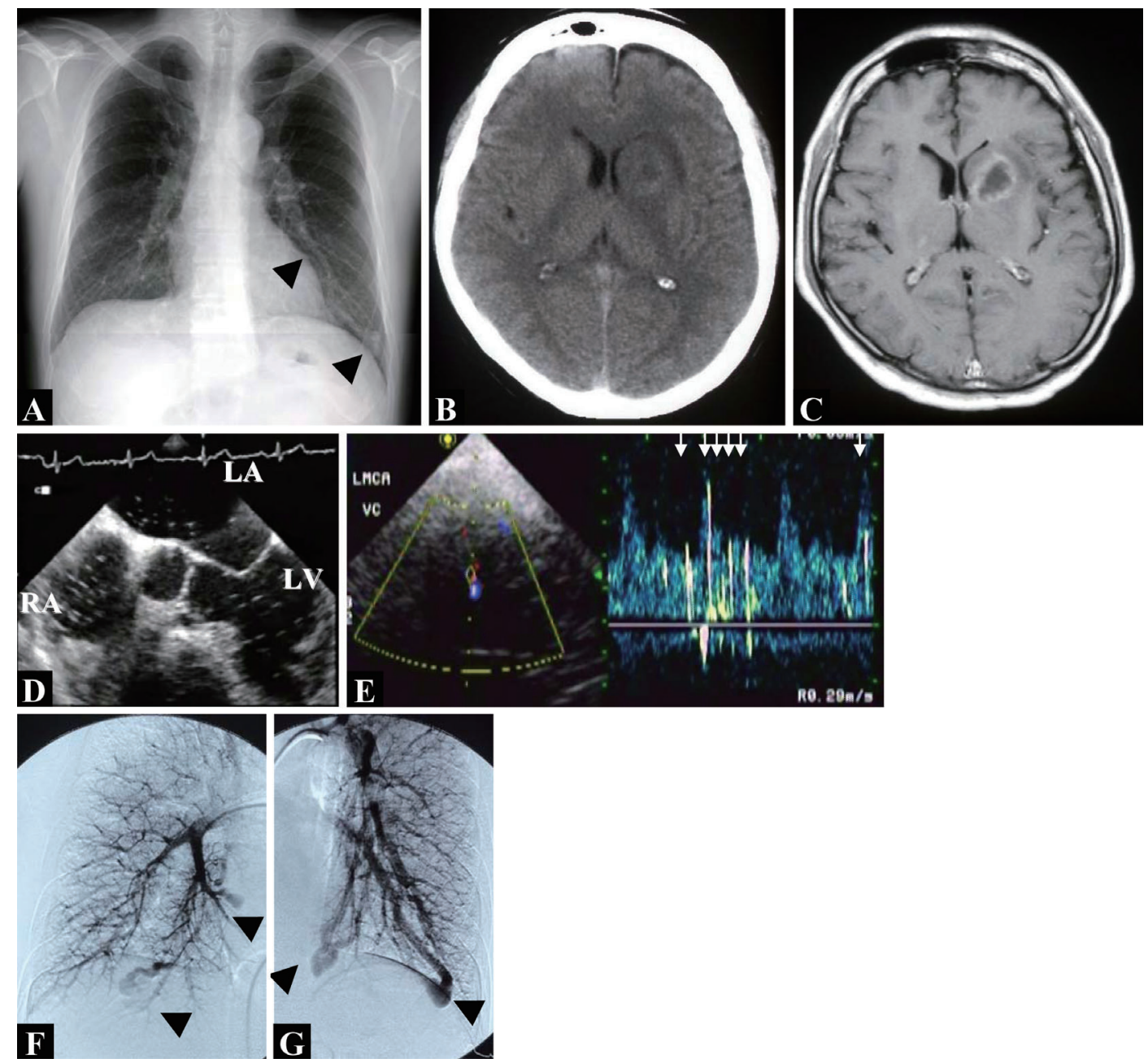

Figure 1. (A) Chest X-ray shows a nodular lesion in the left lower lobe (arrowheads); (B) CT shows a low density area in the left basal ganglia; (C) on this axial contrast enhanced T1-weighted image, the mass has a ring of enhancement; (D) transesophageal echocardiography shows bubbles in the LA after saline contrast medium injection without the Valsalva maneuver; (E) transcranial color Doppler with saline contrast medium shows MES (arrows) from the left middle cerebral artery before embolization therapy. (F) (G) Selective pulmonary angiograms show multiple PAVFs (arrowheads) in the right (F) and left (G) lower lobes. LV: left ventricle, LA: left atrium, RA: right atrium

bubbles in the left atrium without the Valsalva maneuver (Fig. 1D), and the transcranial color Doppler (TCD) showed multiple microembolic signals (MES) without the Valsalva maneuver after injection of agitated saline, indicating the presence of a right-to-left shunt (Fig. 1E). Chest CT with contrast and a pulmonary angiogram showed PAVFs in both lungs (Figs. 1F, 1G). The PAVFs were embolized therapeutically to prevent recurrence of the brain abscess and improve the patient's hypoxia. His hypoxia improved slightly after embolization $\left(\mathrm{PaO}_{2} 76.0\right.$ and $\left.\mathrm{PaCO}_{2} 35.8 \mathrm{mmHg}\right)$.

\section{Discussion}

The present patient had a brain abscess and PAVFs not associated with ROW disease. The results of the arterial blood gases, TEE and TCD suggested the possibility of a right-to-left shunt. The patient was finally diagnosed as hav- ing PAVFs on chest CT with contrast, and this was confirmed by pulmonary artery angiography. The patient had no clinical signs suggestive of ROW disease, such as cyanosis, clubbing, and polycythemia, and no family history of respiratory discomfort, bleeding tendencies, or capillary dilatation. Therefore, the patient was diagnosed as having isolated PAVFs without ROW disease.

There have been some reports of ischemic stroke associated with isolated PAVFs that were detected by TEE and TCD with saline contrast medium (4-6). To the best of our knowledge, however, there have been no reports of isolated PAVFs detected by TEE and TCD that were associated with a brain abscess. In the present patient, TEE and TCD with saline contrast medium revealed MES without the Valsalva maneuver. These results suggested the presence of PAVFs or a large patent foramen ovale. The major finding of this case is that TEE and TCD are very useful for diagnosing PAVFs 
as a cause of brain abscess. In addition, TEE and TCD enabled the speculation of PAVFs without an enhancing agent for CT and pulmonary angiogram. Furthermore, because MES were detected from the left middle cerebral artery which distributed blood flow to the left basal ganglia, the air bubbles injected through the peripheral vein could pass the PAVFs to the location of the brain abscess. The findings might explain that the patient not only had a brain abscess and PAVFs concomitantly, but also PAVFs made septic emboli pass through to the brain and caused the brain abscess, although we could not prove that PAVFs directly caused the brain abscess. A paradoxical embolic mechanism should be included as a cause of not only ischemic stroke but also brain abscess.

The well-known etiologic factors of brain abscess are otitis media, paranasal sinusitis, a past history of craniotomy or open head injury, and cyanotic congenital heart disease. On the other hand, the most prominent complications of PAVFs are neurologic events, including transient ischemic attacks, strokes, and brain abscesses from the right-to-left shunt that bypasses the normal filtering action of the lungs (2). An isolated PAVF without ROW disease could cause paradoxical brain embolism (4-6). In the present patient, the right-to-left pulmonary shunt might have provided access for the septic emboli to bypass the natural pulmonary capillary filter and cause the brain abscess, although there was no evidence of the infectious origin. Streptococcus intermedius is known to be a significant component of the normal oral flora and one of the most common causes of oral and systemic pyogenic infections. However, dental infection is less frequently documented as a predisposing cause of brain abscess (7). Many cases of cryptogenic brain abscess are believed to be secondary to asymptomatic dental foci of infection (7). It might be possible that the right-to-left shunt through the PAVFs allowed passage of paradoxical septic emboli to the cerebral arteries and contributed to the patient's susceptibility to a brain abscess caused by Streptococcus intermedius.

In the present patient, the brain abscess was located in the basal ganglia, although this is a relatively rare location for a brain abscess. Brain abscesses found in the basal ganglia and thalamus have been reported to be associated with congenital heart disease, ROW disease, thoracic infection (8), and patent foramen ovale (9). We hypothesized that the paradoxical septic emboli produced an infarction in the basal ganglia, and that the cerebral hypoxia allowed the bacteria to flourish. PAVF should be included as one of the etiologies of a brain abscess in the basal ganglia. In conclusion, TEE and TCD with saline contrast medium were useful for identifying the presence of a right-to-left shunt in a patient with a brain abscess.

\section{References}

1. Peery WH. Clinical spectrum of hereditary hemorrhagic telangiectasia (Osler-Weber-Rendu Disease). Am J Med 82: 989-997, 1986.

2. Swanson KL, Prakash UB, Stanson AW. Pulmonary arteriovenous fistulas: Mayo Clinic experience, 1982-1997. Mayo Clin Proc 74: 671-680, 1999.

3. Shovlin CL, Jackson JE, Bamford KB, et al. Primary determinants of ischaemic stroke/brain abscess risks are independent of severity of pulmonary arteriovenous malformations in hereditary haemorrhagic telangiectasia. Thorax 63: 259-266, 2008.

4. Kimura K, Minematsu K, Wada K, et al. Transcranial Doppler of a paradoxical brain embolism associated with a pulmonary arteriovenous fistula. AJNR Am J Neuroradiol 20: 1881-1884, 1999.

5. Kimura K, Minematsu K, Nakajima M. Isolated pulmonary arteriovenous fistula without Rendu-Osler-Weber disease as a cause of cryptogenic stroke. J Neurol Neurosurg Psychiatry 75: 311-313, 2004.
6. Todo K, Moriwaki H, Higashi M, Kimura K, Naritomi H. A small pulmonary arteriovenous malformation as a cause of recurrent brain embolism. AJNR Am J Neuroradiol 25: 428-430, 2004.

7. Wispelwey B, Dacey RG Jr, Scheld WM. Brain Abscess. In: Infection of the Central Nervous System. 2nd ed. Scheld WM, Whitley RJ, Durack DT, Eds. Lippincott-Raven Publishers, Philadelphia, 1997: 463-493.

8. Lutz TW, Landolt H, Wasner M, Gratzl O. Diagnosis and management of abscesses in the basal ganglia and thalamus: a survey. Acta Neurochir (Wien) 127: 91-98, 1994.

9. Friedlander RM, Gonzalez RG, Afridi NA, Pfannl R. Case records of the Massachusetts General Hospital. Weekly clinicopathological exercises. Case 16-2003. A 58-year-old woman with left-sided weakness and a right frontal brain mass. N Engl J Med 348: 2125-2132, 2003.

(C) 2009 The Japanese Society of Internal Medicine http://www.naika.or.jp/imindex.html 